\title{
Relevance of Myelography in Diagnosis of Subarachnoid Hematoma in a Rottweiler with Traumatic Cervical Subluxation - Case Report
}

\author{
Robert Cristian PURDOIU ${ }^{1 *}$, Radu LĂCĂTUȘ ${ }^{1}$, Ciprian OBER² ${ }^{2}$ Cosmin PEȘTEAN ${ }^{2}$, Ionel PAPUC ${ }^{1}$ \\ 1) University of Agricultural Sciences and Veterinary Medicine, Faculty of Veterinary Medicine Department \\ of Semiology, Radiology and Medical Imaging, Mănăștur, no. 3-5, Cluj Napoca, Romania; \\ 2) University of Agricultural Sciences and Veterinary Medicine, Faculty of Veterinary Medicine Department \\ of Anesthesiology and Propaedeutic., Mănăștur, no. 3-5, Cluj Napoca, Romania. \\ * Corresponding author: robert.purdoiu@usamvcluj.ro
}

Bulletin UASVM Veterinary Medicine 73(1) / 2016,

Print ISSN 1843-5270; Electronic ISSN 1843-5378

DOI:10.15835/buasvmcn-vm: 11963

\begin{abstract}
Traumatic luxation or subluxation of the cervical vertebrae can cause unstable spinal injuries, such trauma has the potential to disrupt the subarachnoid blood vessel and produce hematoma that will compress the spinal cord. Being located in the soft tissue of the marrow that hematoma will be impossible to examine through conventional radiography.

A 1 year, Rottweiler dog was brought for radiography examination presenting neck pain and tetra paresis. Radiographic exposures were done in lateral and dorso-ventral recumbency to examine the cervical vertebrae. Cervical myelography was done using contrast agent Optiray 350 (Ioversol, manufactured by Mallinckrodt Pharmaceuticals), 0,5 ml/kg. The contrast agent was injected in the cervical area in occipito-athloidian space.

Conventional radiography shows a normal aspect of the cervical vertebrae without any sign of fractures. It was observed a narrowing of the space between occipital and atlas. Puncture of the cervical area of the spine show no cerebrospinal fluid. Injection of the contrast agent was done easily. Myelography was performed at intervals of 2 minutes. Myelography revealed a filling defect in the dorsal part of the marrow caudally of the atlas that corresponded to a hematoma.

Myelography is useful in determining the filling defects due to blood clot formation on the marrow after a posttraumatic event.
\end{abstract}

Keywords: cervical luxation, dog, myelography, subarachnoid hematoma

\section{INTRODUCTION}

Traumatic luxation or subluxation of the cervical vertebrae can cause unstable spinal injuries, such trauma has the potential to disrupt the subarachnoid blood vessel and produce hematoma that will compress the spinal cord (Kinns et al., 2004). Being located in the soft tissue of the marrow that hematoma will be impossible to examine through conventional radiography. Because of the immediate neurological consequences subarachnoid hematoma should be located as soon as possible, for surgical procedure to be applied if necessary (Duval et al., 1996). Myelography is an easy, sensitive and quick method to diagnostic spinal cord compression especially if other methods such as CT or MRI are not available (Hara et al., 1994; McKee et al., 2000; Barone et al., 2002).

\section{AIMS AND OBJECTIVES}

The aim of this case report was to highlight the feeling defects due to subarachnoid hematoma using the myelography as a quick method of diagnostic. 


\section{MATERIALS AND METHODS}

A 1 year, Rottweiler female dog was brought for radiography, clinical examination presented neck pain, neck stiffness and tetra paresis, which indicate a cervical posttraumatic injury.

Radiographic exposures were done in a lateral and dorso-ventral recumbency to examine the cervical vertebrae in order to remove the suspicion of cervical fracture or luxation. On the radiographies the normal aspect and alignment of the cervical vertebrae was visible without displacement or fractures of the vertebral body (fig 1, fig 2).

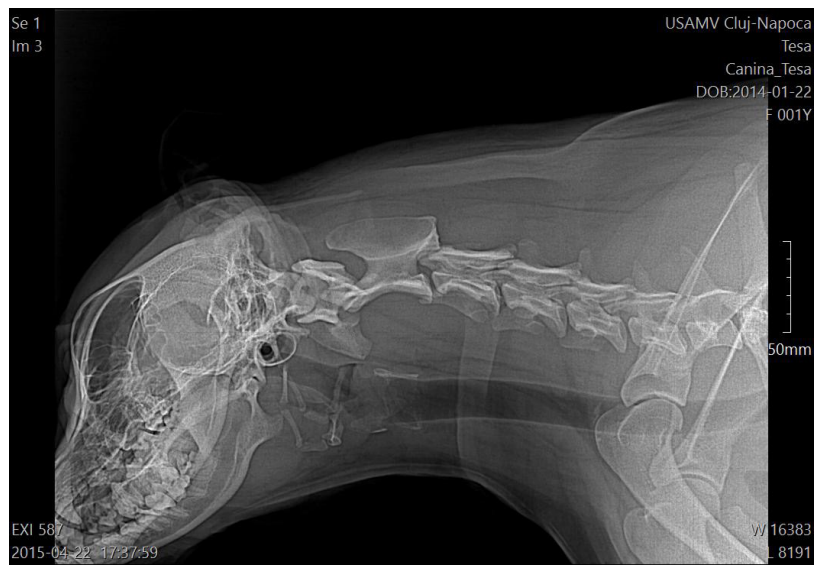

Fig. 1 Lateral exposure of the cervical region, $90 \mathrm{kV}$, $25 \mathrm{mAs}$



Fig. 3 Position of the head during cervical pucture
The dog was prepared for cervical spinal punction, the cervical area was trim and disinfected and the dog was subjected to anesthesia with Propofol 2\% (Fresenius Kabi Deutschland GMBH Germany) administered i.v. until the effects appear. The cervical punction was performed using spinal needle of 20G (fig. 3, fig. 4).

Cervical myelography was done using contrast agent Optiray 350 (Ioversol, manufactured by Mallinckrodt Pharmaceuticals), 0,5 ml/kg. The contrast agent was injected in the cervical area in occipito-athloidian space. After the contrast agent administration, radiography was performed.

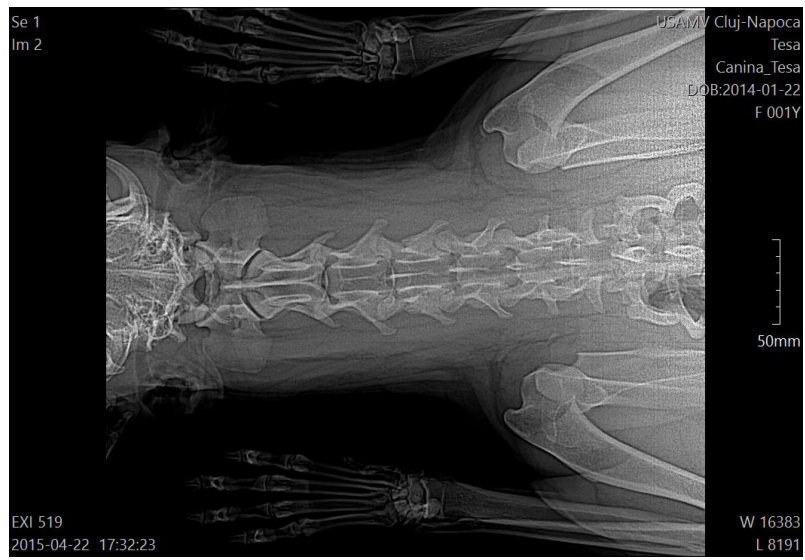

Fig. 2 Dorso-ventral exposure of the cervical vertebrae, $93 \mathrm{kV}, 32 \mathrm{mAs}$

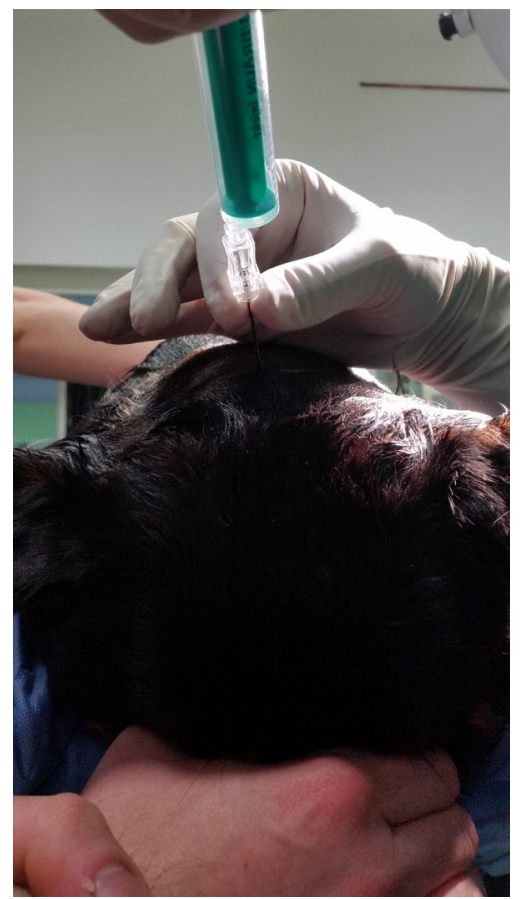

Fig. 4 Cervical pucture 


\section{RESULTS AND DISCUSSION}

Puncture of the cervical area of the spine show a small quantity of cerebrospinal fluid of normal aspect.

Conventional radiography shows a normal aspect of the cervical vertebrae without any sign of fractures or luxation, being observed only a slight narrowing of the space between occipital and atlas.

Injection of the contrast agent was done easily. Myelography was performed at intervals of 2 minutes. Myelography revealed a filling defect in the dorsal part of the marrow caudally of the atlas that corresponded to a hematoma. The contrast agent diffuses both in the cranium highlighting the aspect of the central nervous system but also in the cervical area.

On latero-lateral exposure the filling defect was of $17.79 \mathrm{~mm}$ in length (fig. 5, fig. 6).

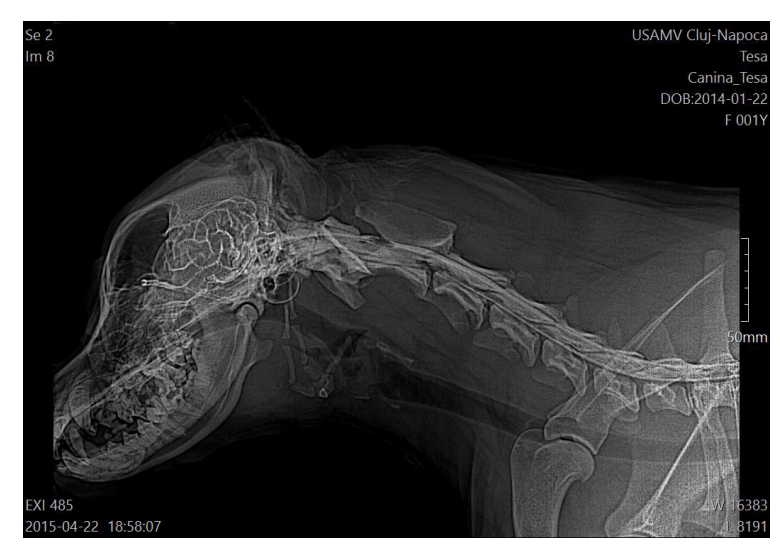

Fig. 5 Highlighting the post contrast aspect of the central nervous system and the filling defect (white arrow)

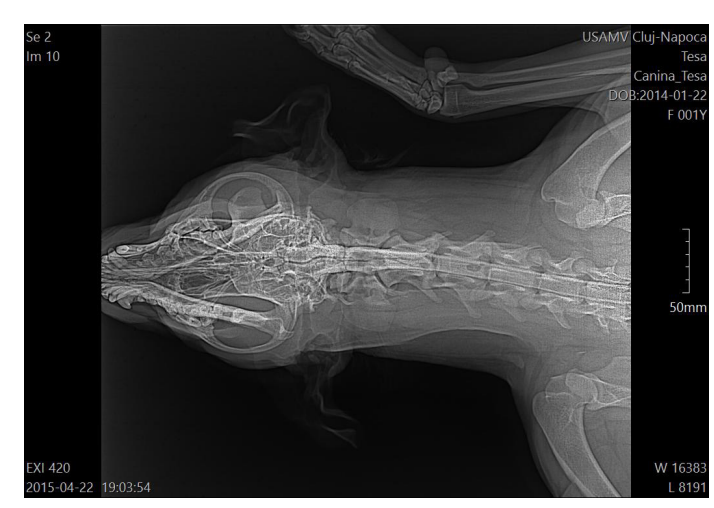

Fig. 7 Dorso-ventral exposure, 90 kV, 25 mAs
Dorso-ventral exposure show another filling defect situated at the $\mathrm{C}_{3}$, which corresponded to a second subarachnoid hematoma, this one couldn't be highlighted on latero-lateral exposure. The size of hematomas in dorso-ventral exposure is of $14.26 \mathrm{~mm} / 31.52 \mathrm{~mm}$ and $9.27 \mathrm{~mm} / 13.45 \mathrm{~mm}$ for the second one (fig. 7, fig. 8).

After the radiological diagnostic the dog was put on proper medication, the symptoms disappearing in approximately 2 weeks.

\section{CONCLUSION}

Myelography is useful in determining the filling defects due to blood clot formation on the marrow after a posttraumatic event. A correct diagnostic prevent unnecessary time delay or an unrequired surgery, through myelography can be highlight the filling defect produced by hematoma or disk protrusion, the vet being able to identify the correct place in case of an intervention.

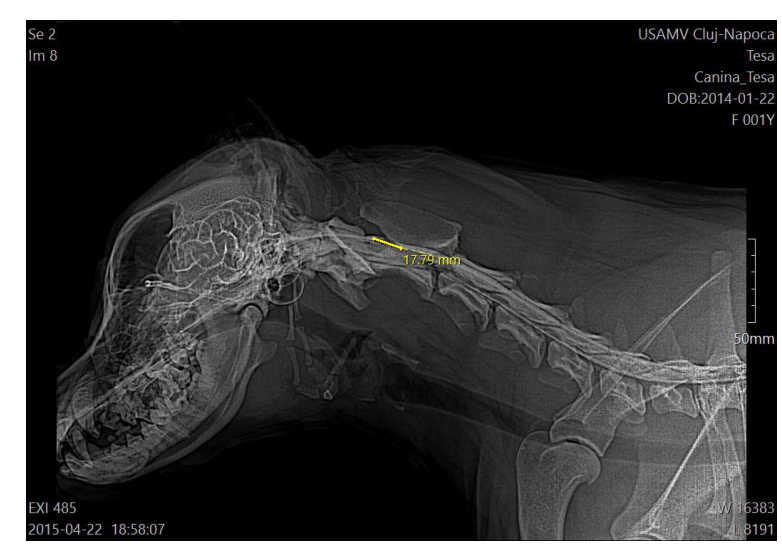

Fig. 6 Position and dimension of the subarachnoid hematoma

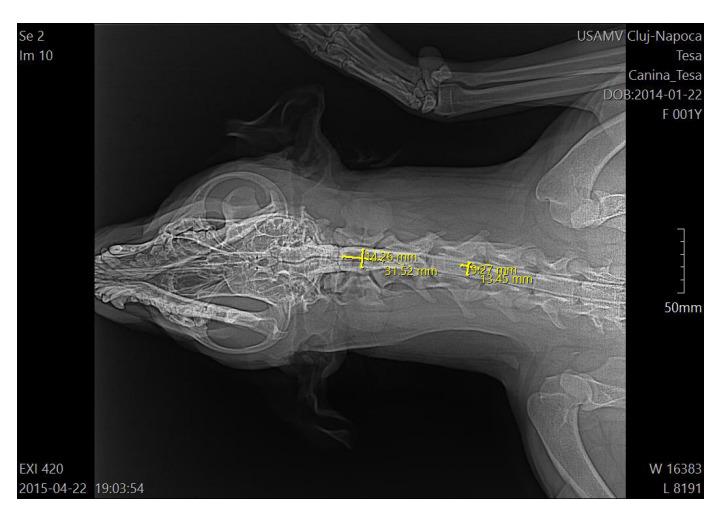

Fig. 8 Size and position of filling defects in dorsoventral eposure 


\section{REFERENCES}

1. Duval J, Dewey C, Roberts R, Aron D (1996). Spinal cord swelling as a myelographic indicator of prognosis: $a$ retrospective study in dogs with intervertebral disc disease and loss of deep pain proprioception. Vet Surg, 25:6-12.

2. Kinns J, Mai W, Seiler G, Zwingenberger A, Johnson V, Cáceres A, Valdés-Martínez A, Schwarz T (2004). Radiographic sensitivity and negative predictive value for acute canine spinal trauma, Veterinary Radiology \& Ultrasound, Volume 47, Issue 6, p. 563-570.
3. Hara Y, Tagawa M, Ejima H (1994). Usefulness of computed tomography after myelography for surgery on dogs with cervical intervertebral disc protrusion. J Vet Med Sci, 56:791-794.

4. McKee WM, Penderis J,Dennis R. (2000). Obstruction of contrast medium flow during cervical myelography. Vet Radiol Ultrasound, 41:342-343.

5. Barone G, Ziemer LS,Schofer FS, Steinberg SA. (2002). Risk factors associated with development of seizures after use of iohexol for myelography in dogs: 182 cases (1998). J Am Vet Med Assoc, 220:1499-1502. 